\section{UNIQUE ORTHODONTIC APPLIANCE}

Once in a while a new product comes along that really makes dentists sit up and take notice and this year that product is the Oralign Ltd orthodontic appliance.

Designed to tackle overcrowding in one or both arches, the Oralign Ltd can be used to straighten the anterior teeth in less than 14 weeks when worn 14 hours a day including overnight. The Oralign Ltd system uses a unique method of interdental stripping with a small discreet removable appliance that doesn't interfere with the rest of the mouth.

During consultation simply take

\section{FIGHT EROSION}

Oral-B's new all-in-one Pro-Expert toothpaste, with its unique combination of polyphosphate and stabilised stannous fluoride, may help reduce the tooth erosion increasingly seen as a result of modern lifestyles.

This new partnership of ingredients in Oral-B Pro-Expert toothpaste has

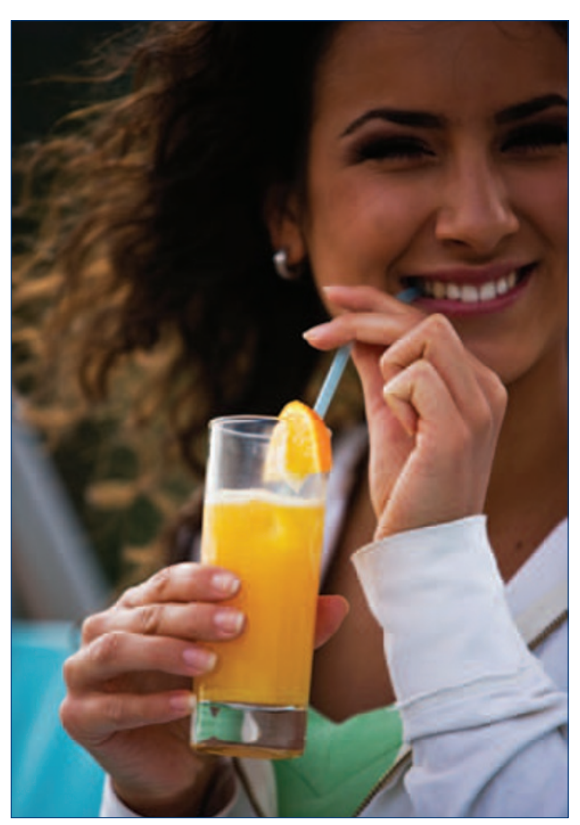

X-rays, photos and full mouth impressions and Oralign Ltd will do the rest. With a full treatment plan and projected outcome provided together with costings, you can offer your patients a satisfactory outcome every time.

With the Oralign Ltd orthodontic appliance, both adult and younger patients can have a straight, beautiful smile in less than four months, making orthodontics available to even the most self conscious of patients.

An ideal source of revenue for every dentist, Oralign Ltd is the ideal orthodontic solution for your practice.

Reader response number 53

created an innovative and comprehensive range of oral health benefits. These include protection against gum problems, plaque, caries, calculus formation, dentinal hypersensitivity, staining and bad breath as well as erosion.

Caused by the increasing consumption of acidic foods and drinks, including fruit juices and soft drinks, tooth erosion is not reversible and can be aesthetically challenging. Clinical and laboratory studies comparing pastes with stabilised stannous fluoride to those without showed that the technology significantly reduced progression of erosion. The effect is due to the creation of a protective stannous containing barrier resistant to acid attacks and protecting the underlying enamel surfaces.

However, of additional benefit to users of Oral-B Pro-Expert toothpaste with its unique combination of polyphosphate and stabilised stannous fluoride is the ability of the latter to reduce plaque. This is achieved by inhibiting bacterial metabolic processes and reducing their ability to adhere to intra-oral surfaces. Reader response number 54

\section{YOUR ORAL HEALTH GURU}

Explaining oral health issues to patients is one of the key drivers in treatment plan acceptance. Now new chairside software guru is helping to make oral health education easy and interactive.

guru is much more than simply a generic educational tool; it enables practitioners to increase treatment plan acceptance by customising presentations through the incorporation of individual patient X-rays, intraoral and extraoral images. In addition, the patient explanation can be augmented by pausing the animation and annotating directly onto the screen via a tablet and stylus, or by recording an audio explanation that addresses the specific needs of the individual case. Presentations can then be printed, emailed or burnt to a DVD or memory stick for the patient to take away.

The guru library, which is constantly being updated and refined, currently contains more than 200 excellent quality 3D animations on a range of treatment options, including an extensive section on oral health.

In addition to its chairside usage, guru can also be employed in the patient waiting area. Playlists featuring oral hygiene tips and techniques as well as treatment options can be displayed on a screen providing an interesting and information backdrop for the practice.

guru provides an excellent, hitech experience for patients wholly familiar with visual media, enabling you to improve patient communication and increase uptake of treatment recommendations.

Reader response number 55

\section{CARIES SALIVA CHECK}

To further enhance GC's Minimal Intervention programme, the company have developed GC Saliva-Check Mutans. GC Saliva-Check Mutans shows whether a patient carries a high level of Streptococcus mutans in the saliva.
This chairside diagnostic tool helps to demonstrate whether a patient is at further risk of caries development, without the need for bacterial cultures. Once diagnosed, the practitioner can act upon this knowledge by prescribing effective oral hygiene measures accordingly.
It takes just 15 minutes to achieve a result with GC Saliva-Check Mutans, which will show the number of colonyforming caries bacteria in the saliva. Perfect for all age groups, GC Salivacheck Mutans helps in the fight against caries development.

Reader response number 56 